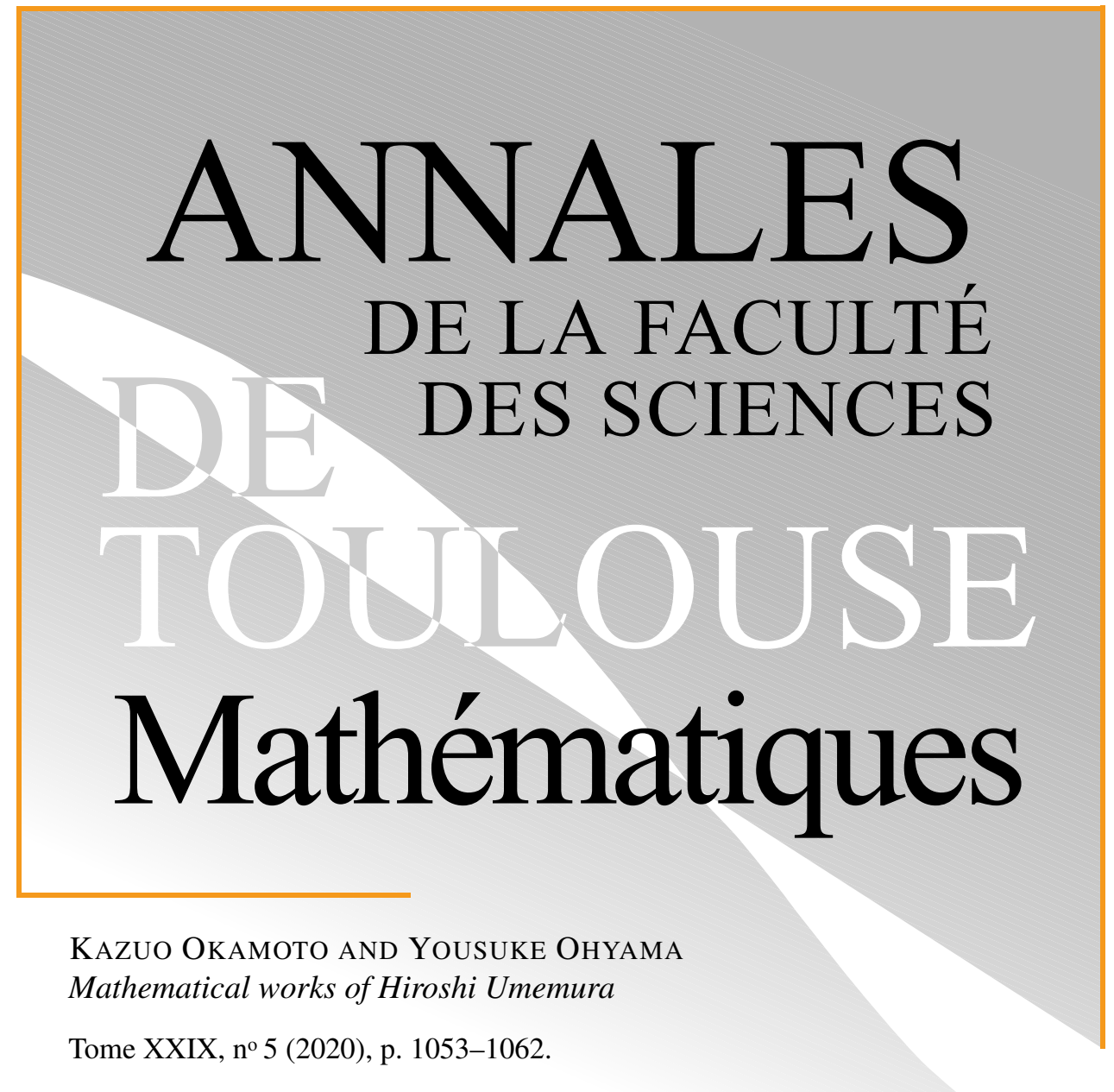

https://doi.org/10.5802/afst.1656

(C) Université Paul Sabatier, Toulouse, 2020.

L'accès aux articles de la revue «Annales de la faculté des sciences de Toulouse Mathématiques » (http://afst.centre-mersenne.org/) implique l'accord avec les conditions générales d'utilisation (http://afst.centre-mersenne.org/legal/). Les articles sont publiés sous la license CC-BY 4.0.

(c) $\underset{B Y}{(1)}$

Publication membre du centre

Mersenne pour l'édition scientifique ouverte MERSENNE http://www.centre-mersenne.org/ 


\title{
Mathematical works of Hiroshi Umemura
}

\author{
KazuO OKamoto $^{(1)}$ And Yousuke Ohyama ${ }^{(2)}$
}

\begin{abstract}
We give an overview of Umemura's mathematical research on algebraic geometry, the Painlevé equations and the Galois differential theory.

RÉsumé. - Nous donnons un aperçu des recherches mathématiques de Hiroshi Umemura sur la géométrie algébrique, les équations de Painlevé et la théorie de Galois différentielle.
\end{abstract}

\section{A short biography of Hiroshi Umemura}

Hiroshi Umemura was born in 1944 at Aichi prefecture in Japan. In 1967 he graduated from Nagoya University, where he spent most of his career. At that time Hisasi Morikawa was a Professor in algebra and Tadao Oda came to Nagoya from the United State in 1968. He went to IHES at Paris in 1971-1972 and stayed at Strasbourg twice, 1975-1976 and 1984-1985.

In the 1970 s he studied algebraic geometry, especially on vector bundles over abelian varieties and maximal connected algebraic groups contained in the Cremona group $\operatorname{Aut}_{k} k\left(x_{1}, x_{2}, \ldots, x_{n}\right)$. Umemura and Mukai [U6] found a new Fano threefold $U_{22}$, which was missed by Fano and Iskovskih. The classification of the Fano varieties were reconsidered after their construction.

When he visited Strasbourg in 1984, he analyzed Painlevé's Leçons de Stockholm [8] in detail and he unveiled Painlevé's theory on irreducibility of nonlinear differential equations. He gave a rigorous definition of classical functions, which are reduced to known functions, such as abelian functions or solutions of linear equations, in terms of the differential Galois theory based on Grothendieck's scheme theory.

(1) National Institution for Academic Degrees and Quality Enhancement of Higher Education, 1-29-1 Gakuen-nishimachi Kodaira-shi, Tokyo 187-8587, Japan

(2) Department of Mathematical Sciences, Tokushima University, 2-1 Minamijyousanjima-cho, Tokushima 770-8506, Japan — ohyama@tokushima-u.ac.jp 
He also gave a precise definition of irreducibility of the Painleve equations based on his theory on classical functions. Any classical solution of the Painlevé equations is either an algebraic solution or a hypergeometric-type solution. A complete classification of all the classical solutions of the Painlevé equations has been achieved by many researchers. Thus the irreducibility of the Painlevé equations of all types are completely shown until 2014.

It might be a dream of Galois and Lie to build a theory on transformation groups of differential equations. In the beginning of the twentieth century, Drach and Vessiot proposed a differential Galois theory, but their theory was not rigorous and difficult to understand. After a fierce struggle against long papers by Vessiot, Umemura constructed an infinite dimensional Galois theory in a rigorous framework. His infinite Galois theory is one of the most successful differential Galois theories which go beyond classical differential Galois theories in finite dimensions, such as the Picard-Vessiot theory and Kolchin's theory. His theory is essentially equivalent to Malgrange's theory on differential Galois groupoids. He tried to determine the differential Galois group of the Painleve equations and gave the Galois group Infgal of the first Painlevé equation under a conjecture.

He received the MSJ Algebra Prize in 1998 for his study of the Painlevé equations and differential Galois theory from the Mathematical Society of Japan. He published 55 papers and two Japanese books. One is a text book [I] on elliptic functions and the other is a monograph [II] on Galois' dream on la théorie de l'ambiguïté. He retired from Nagoya university in 2008 and was bestowed the title of emeritus professor. His theory on the differential Galois group is a monumental work in the study of differential equations and many researchers are studying his infinite Galois theory now.

The authors would like to express our gratitude to Shigeru Mukai for helpful comments and advice.

\section{Works on algebraic geometry}

Umemura started to research algebraic geometry before he became assistant professor in Nagoya University in 1970 . He was thoroughly acquainted with classical literature and reconstructed many modern theories from classical mathematics. He studied Italian classical research on algebraic geometry by Enriques and Fano, who gave a classification of maximal connected algebraic groups contained in the Cremona group $\mathrm{Cr}_{3}$ of three variables. Although their works are difficult to understand and not rigorous from the viewpoint of modern mathematics, Umemura developed a theory of maximal connected algebraic groups contained in $C r_{3}$ [U23, U24, U29]. 
In the 1970s, Iskovskih gave a classification of the Fano threefolds when the second Betti number $b_{2}=1$. One Fano threefold was missed in the list of Fano and Iskovskih. Umemura found a new Fano threefold $U_{22}$, which is the unique minimal model of the quotient of $\mathrm{PGL}_{2}$ by the icosahedral subgroup, with Shigeru Mukai [U6]. Before the construction by Mukai and Umemura, double projection from a line with normal bundle $\mathcal{O} \oplus \mathcal{O}(-1)$ was a standard tool to study Fano threefolds. But the new one has no such a line on it. After reexamination, the classification of Fano threefolds became complete with adding $U_{22}$.

One of his famous work is the appendix [U26] in Mumford's Tata Lectures on Theta II. Abel proved that quintic equations cannot be solved by radicals. Hermite and Kronecker showed that quintic equations can be solved by elliptic modular functions. Moreover Jordan proved that algebraic equations of any degree can be solved by modular forms and Thomae gave a clear formula of solutions. In [U26], Umemura explained how to solve algebraic equations by Siegel modular forms. This appendix has been a standard reference on resolution of algebraic equations.

Around 1983, he had an interest on Painlevé's works on the birational automorphism groups of algebraic surfaces in [8, 9]. In [U25], he found a cubic threefold with an infinite dimensional birational automorphism group, which contains no algebraic subgroup of positive dimensions. The paper [U25] is the first paper in which Umemura referred to Painlevé's work, but it seems that he had no interest on Painlevé's works on differential equations at this stage. Leçons de Stockholm was known by Japanese school of algebraic geometry in the 1960s and Umemura studied Painlevé's classical research on algebraic surfaces in his study on birational automorphism groups of algebraic threefolds. Painlevé's Stockholm Leçons was also referred to by the Italian school, for example in [2].

\section{Works on differential Galois theory and the Painlevé equations}

When Ellis Kolchin visited Nagoya in 1970, Umemura was a graduate student and picked up Kolchin from his hotel to the University. Umemura said to Kolchin that he would go to Strasbourg to study under Pierre Cartier. He never considered that he would study the differential Galois theory in the future even when he met Kolchin. Umemura visited Strasbourg in 1971, but he soon moved to IHES with Cartier.

In 1983, Jean-François Pommaret visited Japan with his new book [11]. He also gave a talk on his differential Galois theory at Nagoya. Umemura said 
"I cannot understand Pommaret's talk. But he taught me many researches on the differential Galois theory since Lie, Picard, Drach and Vessiot. I was excited over the Galois theory on differential equations." But even when Umemura met Pommaret, he did not consider that he would study the differential Galois theory later.

When Umemura went to Strasbourg in 1984, he investigated minutely Painlevé's Leçons de Stockholm [8]. He realized that Painlevé understood irreducibility of differential equations very well but Painlevé did not have a mathematical language to express it. Umemura started to study irreducibility of the Painleve equations and the differential Galois theory. He gave a talk [U27] at un colloque Franco-Japonais "Équations différentielles dans le champ complexe" at Strasbourg in 1985. He published two long papers in 1990. One [U36] is on irreducibility of the first Painlevé equation and the second one [U35] is on the differential Galois theory. When he wrote a short survey [U28] on works of Fano in 1885, he pointed out that Fano studied not only algebraic geometry but also linear differential equations and the Picard-Vessiot theory.

Painlevé himself gave a proof on irreducibility of the first Painlevé equation, but his proof was far from complete. When Painlevé has 'shown' irreducibility [10], it caused a dispute against R. Liouville. Painlevé's proof is based on Drach's theory [3], but Drach's theory is a kind of infinite dimensional differential Galois theory and it was difficult to construct a rigorous theory on infinite dimensional group theory around 1900.

Umemura considered what is a "known function" by analyzing Leçons de Stockholm. In order to give a precise definition of irreducibility, we need to give a definition of known functions. He gave a definition of classical functions, which are reduced to elementary functions, abelian functions and solutions of linear differential equations, in terms of the differential Galois theory. For a differential field extension by classical functions, the differential Galois group is an algebraic group. By Chevalley's theorem, any algebraic group is an extension of an abelian variety by a linear algebraic group. Since Umemura's classical functions contain abelian functions, Umemura's first theory [U35] goes beyond the Picard-Vessiot theory which treats only linear differential equations, but it is still finite-dimensional like Kolchin's theory.

Based on rigorous theory on the differential Galois groups, he gave a modern definition of irreducibility of the Painlevé equations [U32]. In 1987, K. Nishioka [7] also gave irreducibility of the first Painlevé equation independently. Nishioka's proof does not depend on infinite dimensional differential Galois theory, contrary to Painlevé's guess. 
There exist two types of classical solutions of the Painlevé equations. One is algebraic functions. Let $K$ be a field of rational functions. For any algebraic solution $y$, the transcendence degree tr.d $[K\langle y\rangle ; K]=0$. The second is classical transcendental solutions. Any classical transcendental solution $y$ satisfies a Riccati equation and tr.d $[K\langle y\rangle ; K]=1$. When tr.d $[K\langle y\rangle ; K]=2$, $y$ is not a classical function (irreducibility). The irreducibility of the Painlevé equations is a good testing ground to apply the differential Galois theory.

Umemura's strategy to show the irreducibility of the Painlevé equations is as follows:

- Classify all algebraic solutions.

- Find invariant polynomials in the initial value spaces.

We explain invariant polynomials in the case of the first Painlevé equation. We take a differential field $K=(K, \delta)$, an extension of $(\mathbb{C}(t), d / d t)$. We consider a polynomial ring $K[p, q]$. We take a vector field $X=\delta+p \partial / \partial q+$ $\left(6 q^{2}+t\right) \partial / \partial p$. If there exist $G \in K[p, q]$ such that $X F=G F$, the polynomial $F \in K[p, q]$ is called $X$-invariant. Moreover, when $F \notin K, F$ is called $a$ nontrivial $X$-invariant polynomial. Geometrically, $X$ is a vector field on the affine space and the curve $F=0$ is tangent to $X$. We can define an invariant polynomial for other Painlevé equations.

When any $X$-invariant polynomial is trivial, Umemura [U36] said that a "condition $(\mathrm{J})$ " is satisfied. He found an algorithm to determine an invariant polynomial [U41], and proved irreducibility of the Painlevé equations of higher type with $\mathrm{H}$. Watanabe. The notation $(\mathrm{J})$ means the initial of the Japanese word "Joken", which means "condition". This is a joke by Umemura!

He also studied rational solutions of the fifth and sixth Painlevé equations [U44]. The corresponding $\tau$ functions are called the Umemura polynomials. Since his first announcement [U42] on the Umemura polynomial for the sixth Painlevé equation was not in print, we publish it in this volume (see section 4).

Umemura made the most of opportunity that the Painlevé equations are actively studied in Japan. Moreover, Georges Reeb in Strasbourg considered that the Painlevé equations are pioneer works of foliations, and he edited three volumes of Oeuvres de Paul Painlevé in 1972-1975 (K. O. also stayed at Strasbourg and assisted editorial work).

In the 1990s, Umemura studied a theory of differential Galois groups in infinite dimensions. He always carried papers by Vessiot $[12,13,14,15]$ for many years. Drach and Vessiot considered differential Galois theory for nonlinear differential equations, but their theory is far from rigorous. Umemura 
finally constructed his infinite differential Galois theory [U40, U41, U43], but few researchers had an interest on his theory at first. In 1995, he gave a talk at Colloque Trajectorien à la mémoire de Georges Reeb et Jean-Louis Callot at Strasbourg-Obernai (The proceeding is [4]) and André Lichnerowicz appreciated his talk. He also gave a talk [U45] at Kyoto in 1999 and Bernard Malgrange had a deep interest in Umemura's theory. Malgrange constructed his theory on Galois groupoids in 2001 [6].

Umemura's theory is one of the most successful theoris on the differential Galois groups. It might be Galois' dream on "la théorie de l'ambiguitée". His infinite Galois theory goes beyond classical differential Galois theory by Kolchin. Although Umemura's theory looks different from Malgrange's theory on the differential Galois groupoids, both are coincident with each other in many examples. For example, Umemura explained the equivalence in the absolute case [U52] where the base field consists of only constants. He also tried to connect his theory to Malgrange's theory on differential Galois groupoids over a general base field, but this work was not finished.

Umemura, Pommaret and Malgrange met at IHES in the fall 2010. Three researchers have their own differential Galois theory. After a stormy discussion, Umemura and Pommaret did not achieve consensus on differential Galois theory. This is the last time that Umemura met Pommaret. Umemura met Anand Pillay at the same time and he appreciated a talk by Pillay.

He continued to apply his infinite Galois theory to other equations such as soliton equations or difference equations. See [5] for a unification of the Taylor morphism with the Euler morphism of Morikawa and Umemura [U5].

It is a difficult problem to determine infinite Galois groups. Umemura tried to determine the Galois group Inf-gal $(L / K)$ of the first Painlevé equation with Daniel Bertrand [U1] and he succeeded partly in [U48]. See [1] for a computation of the Malgrange groupoid of the Picard-Painlevé equation, inspired by Umemura's study of classical functions. Thus Umemura's research has influenced younger generation. He proposed many unsolved questions in his talk [U49] at Angers. They would be future works for us, successors of Hiroshi Umemura.

\section{Unpublished paper on special polynomials associated with the Painlevé equations}

This volume contains an unpublished paper [U42] by Umemura. Umemura gave a talk at the conference "Theory of nonlinear special functions: the Painlevé transcendents" held at Montreal in 1996, but the proceeding is 
not published. Umemura showed a series of rational solutions of the sixth Painlevé equations. The $\tau$-function of the rational solution is now called the Umemura polynomial, which has a rich structure related to the Young diagram. The first half of [U42] is a good survey of special solutions of Painlevé equations.

Some researches might wonder why the title of the paper [U7] is part II without part I, but [U42] shows that Umemura did write part I. One of the authors (Y. O.) received a copy of the paper [U42] from Umemura himself and it is our pleasure to present in this memorial issue. We express special thanks to Mrs. Yuko Fujii, a secretary in Tokushima University, for typesetting the manuscript in $\mathrm{T}_{\mathrm{E} X}$.

\section{List of Umemura's works}

\section{Published papers}

[U1] D. Bertrand \& H. Umemura, "On the definitions of the Painlevé equations", RIMS Kokyuroku 1296 (2002), p. 29-34.

[U2] S. Fukutani, K. Окамото \& H. Umemura, "Special polynomials and the Hirota bilinear relations of the second and the fourth Painlevé equations", Nagoya Math. J. 159 (2000), p. 179-200.

[U3] A. Masuoka, K. Saito \& H. Umemura, "Toward quantization of Galois theory", Ann. Fac. Sci. Toulouse, Math. 29 (2020), no. 5, p. 1319-1431.

[U4] S. Morikawa, K. Saito, T. Takeuchi \& H. Umemura, "Discrete Burgers' equation, binomial coefficients and mandala", Math. Comput. Sci. 4 (2010), no. 2-3, p. 151-167.

[U5] S. Morikawa \& H. Umemura, "On a general difference Galois theory. II", Ann. Inst. Fourier 59 (2009), no. 7, p. 2733-2771.

[U6] S. Mukai \& H. Umemura, "Minimal rational threefolds", in Algebraic geometry (Tokyo and Kyoto 1982), Lecture Notes in Mathematics, vol. 1016, Springer, 1983, p. $490-518$.

[U7] M. Noumi, S. Okada, K. Okamoto \& H. Umemura, "Special polynomials associated with the Painlevé equations. II", in Integrable systems and algebraic geometry, World Scientific, 1997, p. 349-372.

[U8] K. Saito \& H. Umemura, "Can we quantize Galois theory?", in Proceedings of Various aspects of the Painlevé equations, to appear.

[U9] M.-H. Saito \& H. Umemura, "Painlevé equations and deformations of rational surfaces with rational double points", in Physics and combinatorics (Nagoya, 1999), World Scientific, 2001, p. 320-365.

[U10] H. Umemura, "Formal moduli for p-divisible groups", Nagoya Math. J. 42 (1971), p. 1-7.

[U11] - "Dimension cohomologique des groupes algébriques commutatifs", Ann. Sci. Éc. Norm. Supér. 5 (1972), p. 265-276.

[U12] - "Fibrés vectoriels positifs sur une courbe elliptique", Bull. Soc. Math. Fr. 100 (1972), p. 431-433.

[U13] — "La dimension cohomologique des surfaces algébriques", Nagoya Math. J. 47 (1972), p. 155-160. 
[U14] - "Cohomological dimension of group schemes", Nagoya Math. J. 52 (1973), p. $47-52$.

[U15] - "Some results in the theory of vector bundles", Nagoya Math. J. 52 (1973), p. $97-128$.

[U16] - "A theorem of Matsushima", Nagoya Math. J. 54 (1974), p. 123-134.

[U17] - "Stable vector bundles with numerically trivial Chern classes over a hyperelliptic surface", Nagoya Math. J. 59 (1975), p. 107-134.

[U18] — "On a certain type of vector bundles over an Abelian variety", Nagoya Math. J. 64 (1976), p. 31-45.

[U19] — , "On a property of symmetric products of a curve of genus 2", in Proceedings of the International Symposium on Algebraic Geometry (Kyoto, 1977), Kinokuniya Book-Store, 1977, p. 709-721.

[U20] - "On a theorem of Ramanan", Nagoya Math. J. 69 (1978), p. 131-138.

[U21] - "Moduli spaces of the stable vector bundles over abelian surfaces", Nagoya Math. J. 77 (1980), p. 47-60.

[U22] — "Sur les sous-groupes algébriques primitifs du groupe de Cremona à trois variables", Nagoya Math. J. 79 (1980), p. 47-67.

[U23] - "Maximal algebraic subgroups of the Cremona group of three variables. Imprimitive algebraic subgroups of exceptional type", Nagoya Math. J. 87 (1982), p. 59-78.

[U24] - "On the maximal connected algebraic subgroups of the Cremona group. I", Nagoya Math. J. 88 (1982), p. 213-246.

[U25] - "Algebro-geometric problems arising from Painlevé's works", in Algebraic and Topological Theories -to the memory of Dr. Takehiko MIYATA, Kinokuniya Company Ltd, 1984, p. 467-495.

[U26] - "Resolutions of algebraic equations by theta constants", in Tata Lectures on Theta II, Progress in Mathematics, vol. 43, Birkhäuser, 1984.

[U27] - "Birational automorphism groups and differential equations", in Équations différentielles dans le champ complexe, Vol. II (Strasbourg, 1985), Publ. Inst. Rech. Math. Av., Univ. Louis Pasteur, 1985, p. 119-227.

[U28] — "Gino Fano", Sügaku 37 (1985), p. 169-178.

[U29] - On the maximal connected algebraic subgroups of the Cremona group. II, Advanced Studies in Pure Mathematics, vol. 6, North-Holland, 1985.

[U30] - "Minimal rational threefolds. II", Nagoya Math. J. 110 (1988), p. 15-80.

[U31] - "On the irreducibility of Painlevé differential equations", Sūgaku 40 (1988), no. 1, p. 47-61, translated in Sugaku Expositions, 2 (1989), p. 231-252.

[U32] - "On the irreducibility of the first differential equation of Painlevé", in Algebraic geometry and commutative algebra, Vol. II, vol. 771, Konokuniya Company Ltd, 1988, p. 771-789.

[U33] — "On classical numbers", Sūgaku 41 (1989), p. 1-15, translated in Sugaku Expositions 4 (1991), p. 1-20.

[U34] - "On the Lie-Drach-Vessiot theory", in Proceeding of symposium on algebraic geometry, 1989, p. 173-198.

[U35] - "Birational automorphism groups and differential equations", Nagoya Math. J. 119 (1990), p. 1-80.

[U36] - "Second proof of the irreducibility of the first differential equation of Painlevé", Nagoya Math. J. 117 (1990), p. 125-171.

[U37] — "Classical solutions to Painlevé equations", RIMS Kokyuroku 857 (1994), p. $63-80$.

[U38] - "On a class of numbers generated by differential equations related with algebraic groups", Nagoya Math. J. 133 (1994), p. 1-55. 
[U39] - "The Painlevé equation and classical functions", Sūgaku 47 (1995), p. 341359, translated in Sugaku Expositions 11 (1998), p. 77-100.

[U40] - "Differential Galois theory of infinite dimension", Nagoya Math. J. 144 (1996), p. 59-135.

[U41] , "Galois theory of algebraic and differential equations", Nagoya Math. J. 144 (1996), p. 1-58.

[U42] - "Special polynomials associated with the Painlevé equations. I", Ann. Fac. Sci. Toulouse, Math. 29 (1996), no. 5, p. 1063-1089, talks in Theory of nonlinear special functions: the Painlevé transcendents.

[U43] — "Lie-Drach-Vessiot theory-infinite-dimensional differential Galois theory", in CR-geometry and overdetermined systems (Osaka, 1994), Advanced Studies in Pure Mathematics, vol. 25, 1997, p. 364-385.

[U44] - "100 years of the Painlevé equation", Sūgaku 51 (1999), p. 395-420.

[U45] — "Lie-Drach-Vessiot theory and Painlevé equations", RIMS Kokyuroku 1150 (2000), p. 63-69.

[U46] , "On the transformation group of the second Painlevé equation", Nagoya Math. J. 157 (2000), p. 15-46.

[U47] , "On the definitions of the Painlevé equations", in Proceeding of symposium on algebraic geometry, 2002, p. 76-83.

[U48] — "Monodromy preserving deformation and differential Galois group. I", in Analyse complexe, systèmes dynamiques, sommabilité des séries divergentes et théories galoisiennes. I, Astérisque, vol. 296, Société Mathématique de France, 2004, p. 253-269.

[U49] - "Galois theory and Painlevé equations", in Théories asymptotiques et équations de Painlevé, Séminaires et Congrès, vol. 14, Société Mathématique de France, 2006, p. 299-339.

[U50] - "Invitation to Galois theory", in Differential equations and quantum groups, IRMA Lectures in Mathematics and Theoretical Physics, vol. 9, European Mathematical Society, 2007, p. 269-289.

[U51] - "Sur l'équivalence des théories de Galois différentielles générales", $C . R$. Math. Acad. Sci. Paris 346 (2008), p. 1155-1158.

[U52] - "On the definition of the Galois groupoid", in Differential equations and singularities, Astérisque, vol. 323, 2009, p. 441-452.

[U53] - "Picard-Vessiot theory in general Galois theory", in Algebraic methods in dynamical systems, Banach Center Publications, vol. 94, Polish Academy of Sciences, 2011, p. 263-293.

[U54] H. Umemura \& H. Watanabe, "Solutions of the second and fourth Painlevé equations. I", Nagoya Math. J. 148 (1997), p. 151-198.

[U55] - "Solutions of the third Painlevé equation. I", Nagoya Math. J. 151 (1998), p. $1-24$.

\section{Books published in Japan}

[I] H. Umemura, Theory on elliptic functions, University of Tokyo Press, 2000.

[II] — Galois: la magnifique théorie de l'ambiguïté, Gendai-Sugakusha, 2011. 


\section{Bibliography}

[1] G. Casale, "The Galois groupoid of Picard-Painlevé VI equation", RIMS Kôkyûroku Bessatsu B2 (2007), p. 15-20.

[2] G. Castelnuovo \& F. Enriques, "Grundeigenschaften der Algebraischen Flächen", Encyklopädie der mathematischen Wissenschaften mit Einschluss ihrer Anwendungen 2 (1915), no. 1, p. 744-755.

[3] J. Drach, "Essai sur une théorie générale de l'intégration et sur la classification des transcendantes", Ann. Sci. Éc. Norm. Supér. (1898), p. 243-384.

[4] A. Fruchard \& A. Troesch (eds.), Colloque trajectorien à la mémoire de Georges Reeb et Jean-Louis Callot (Strasbourg-Obernai, 1995), IRMA et C.N.R.S, 1995.

[5] F. HeIDERICH, "Introduction to the Galois theory of Artinian simple module algebras", in Geometric and differential Galois theories, Séminaires et Congrès, vol. 27, Société Mathématique de France, 2013, p. 69-92.

[6] B. Malgrange, "Le groupoïde de Galois d'un feuilletage", in Essays on geometry and related topics, Monographies de l'Enseignement Mathématique, vol. 2, L'Enseignement Mathématique, 2001, p. 465-501.

[7] K. Nishioka, "A note on the transcendency of Painlevé's first transcendent", Nagoya Math. J. 109 (1988), p. 63-67.

[8] P. PAInlevÉ, Leçons sur la théorie analytique des équations différentielles, professées à Stockholm (1895), Hermann, 1897 (Oeuvres I, pp. 199-807).

[9] - Analyse des Travaux Scientifiques Jusqu'en 1900, Blanchard, 1900 (Oeuvres I, p. $75-196)$.

[10] - "Sur l'irréductibilité des transcendantes uniformes définies par les équations différentielles du second ordre", C. R. Math. Acad. Sci. Paris 135 (1902), p. 411-415.

[11] J.-F. Pommaret, Differential Galois Theory, Mathematics and its Applications, vol. 15, Gordon and Breach Science Publishers, 1983.

[12] E. Vessiot, "Sur la théorie des groupes continus", Ann. Sci. Éc. Norm. Supér. 20 (1903), p. 411-451.

[13] - "Sur la théorie de Galois et ses diverses généralisations", Ann. Sci. Éc. Norm. Supér. 21 (1904), p. 9-85.

[14] - "Sur l'intégration des systèmes différentiels qui admettent des groupes continus de transformations", Acta Math. 28 (1904), p. 307-349.

[15] — "Sur une théorie générale de la réductibilité des équations et systèmes d'équations finies ou différentielles", Ann. Sci. Éc. Norm. Supér. 63 (1946), p. 1-22. 